\title{
Black hole polarization and new entropy bounds
}

\author{
Jacob D. Bekenstein* and Avraham E. Mayo ${ }^{\dagger}$ \\ The Racah Institute of Physics, Hebrew University of Jerusalem, \\ Givat Ram, Jerusalem 91904, Israel
}

(June 6, 2021)

\begin{abstract}
Zaslavskii has suggested how to tighten Bekenstein's bound on entropy when the object is electrically charged. Recently Hod has provided a second tighter version of the bound applicable when the object is rotating. Here we derive Zaslavskii's optimized bound by considering the accretion of an ordinary charged object by a black hole. The force originating from the polarization of the black hole by a nearby charge is central to the derivation of the bound from the generalized second law. We also conjecture an entropy bound for charged rotating objects, a synthesis of Zaslavskii's and Hod's. On the basis of the no hair principle for black holes, we show that this last bound cannot be tightened further in a generic way by knowledge of "global" conserved charges, e.g., baryon number, which may be borne by the object.
\end{abstract}

\section{INTRODUCTION}

A universal bound on the entropy of a macroscopic object of maximal radius $R$ bearing energy $E$ has been proposed by one of us [1]:

$$
S \leq 2 \pi E R / \hbar
$$

*Electronic mail: bekenste@vms.huji.ac.il

†Electronic mail: Mayo@venus.fiz.huji.ac.il 
(units with $G=c=1$ are used). This bound was first inferred by considering the infall of the relevant object into a black hole, and arranging for the infall conditions to cause a minimum of horizon area growth. Appealing to the generalized second law (GSL) [2] then gave bound (1.1) as a condition for the overall entropy not to decrease. This derivation was criticized [3] for leaving out the effects of buoyancy in the acceleration (Unruh) radiation. In some scenarios this makes a difference in the energy that is added to the black hole by the infall, and thus to its horizon area increase. However, it has become clear [4, 包 that if the Unruh acceleration buoyancy comes from a small number of particle species (as it must in our universe), then for objects which are not too thin in one of their dimensions, and whose parts are described by quantum mechanics, the buoyancy correction is indeed negligible, and one can derive bound (1.1) by appealing to the GSL.

Independent support exists for bound (1.1). It is satisfied trivially for composites of nonrelativistic particles by virtue of the fact that entropy of a system is never far from the number of particles involved. And for free massless quantum fields enclosed in volumes of various shapes, the bound's validity has been checked directly. Both numerical verification [6] and analytical proof [7] exist (see review by Bekenstein and Schiffer [8]). The entropy bound can also be inferred directly from the properties of the acceleration radiation [9].

Regarding self-gravitating systems, Sorkin, Wald and Jiu [10] gave evidence that bound (1.1) is valid for thermal radiation on the verge of gravitational collapse, while Zaslavskii [11] proves the bound for a system consisting of a static black hole in equilibrium with thermal radiation in a box. When the black hole has charge $e$, Zaslavskii [12] infers the tighter bound

$$
S \leq \frac{2 \pi E R}{\hbar}\left(1-\frac{e^{2}}{2 E R}\right)
$$

although he admits to some uncertainty regarding the numerical coefficients. Zaslavskii does not claim this form of the bound for systems not containing a black hole.

In its original form (1.1), the entropy bound is saturated by the Schwarzschild black hole [whose entropy is $4 \pi(2 M)^{2} / 4 \hbar=2 \pi M \cdot(2 M) / \hbar$ ]. This prompted the observation [1] that the Schwarzschild black hole is the most entropic object for given size and energy. But 
the Kerr black hole's entropy falls below bound (1.1) (this will be true for any reasonable interpretation of the radius $R$ of the nonspherical Kerr hole).

This asymmetric state of affairs motivated Hod [13] to search for a tighter bound on entropy for objects with angular momentum which is saturated by the Kerr hole. Hod repeats Bekenstein's derivation [14,15] of the minimal increment in Kerr-Newman (KN) horizon area that is caused by an object's infall. That derivation applied the idea of Christodoulou [16] together with Carter's [17,18] integrals of the Lorentz equations of motion to a particle of rest mass $\mu$ and radius $R$ moving in a KN background. The minimal growth in horizon area was found from the conservation laws and the relation they establish between the change in black hole parameters and the energy and orbital angular momentum of a particle in an orbit such that the particle's center of mass (CM) can get to distance $R$ from the horizon:

$$
(\Delta A)_{\min }=8 \pi \mu R
$$

It is remarkable that this minimal area growth is independent of the black hole parameters. Because $\mu$ can be identified with the total proper energy of the object, bound (1.1) follows from (1.3) and the GSL.

Spin of the particle was not taken into account in Carter's integrals. Hod refers instead to Hojman and Hojman's [19] integrals of motion for a neutral object with spin $s$ moving on a KN background. Repeating the argument that led to Eq.(1.3), Hod gets

$$
(\Delta A)_{\min }=8 \pi \mu R\left(1-s^{2} \mu^{-2} R^{-2}\right)^{1 / 2}
$$

Appeal to the GSL then allows Hod to infer

$$
S \leq \frac{2 \pi E R}{\hbar}\left(1-\frac{s^{2}}{E^{2} R^{2}}\right)^{1 / 2}
$$

As Hod remarks, a Kerr black hole of mass $E=m$ and spin $s=j \leq m$ exactly saturates bound (1.5) provided one identifies $R$ with $\left(r_{+}^{2}+j^{2} / m^{2}\right)^{1 / 2}$, where $r_{+}=m+\left(m^{2}-j^{2} / m^{2}\right)^{1 / 2}$ is the radial Boyer-Lindquist coordinate for the Kerr horizon. The identification is reasonable because $4 \pi\left(r_{+}^{2}+j^{2} / m^{2}\right)$ is exactly the area of the Kerr horizon. 
In Sec.II we take up the question of how to derive bound (1.2) for an ordinary charged object (not a system including a black hole as in Zaslavskii [12]) by analogy with the original derivation of bound (1.1) using the GSL. In Sec.III we calculate the change in horizon area that results from lowering a charged object into an electrically isolated black hole, and thus furnish a derivation from the GSL of Zaslavskii's bound (1.2). Sec.IV contains a variant using an electrically grounded black hole; it leads to the same result. In Sec.V we conjecture an entropy bound for rotating charged objects, assemble supporting evidence, and also give a partial proof that it cannot generically be made tighter by taking other conserved quantities, e.g. baryon number, into account.

\section{THE ROLE OF BLACK HOLE POLARIZATION}

Granted the validity of the original entropy bound (1.1) for a macroscopic object, is Hod's bound reasonable from a mundane point of view ? For small $s$ (nonrelativistic rotation) we may expand the r.h.s. of Eq.(1.5) to get

$$
S \leq \frac{2 \pi R}{\hbar}\left[E-\frac{s^{2}}{2 \mu R^{2}}\right]+O\left(s^{4}\right)
$$

where we have replaced $E \rightarrow \mu$ (rest mass) in the denominator. Now an object with moment of inertia $I$ has internal energy $\epsilon=E-s^{2} / 2 I$. For a thin spherical shell $I$ reaches its maximum, $\frac{2}{3} \mu R^{2}$, so that the internal energy for given $E$ and $R$ is also maximized: $\epsilon_{\max }=$ $E-3 s^{2} /\left(4 \mu R^{2}\right)$. The phase space available to the object's degrees of freedom is controlled by $\epsilon$. Hence, if bound (1.1) is valid at $s=0$, we would infer $S \leq(2 \pi R / \hbar)\left[E-3 s^{2} /\left(4 \mu R^{2}\right)\right]$ when $s \neq 0$. Hod's bound is a bit more liberal; as a result, it manages to encompass the Kerr black hole.

Now for a nonrotating object of mass $\mu$, radius $R$ and charge $e$, the Coulomb energy attains its minimum, $e^{2} / 2 R$, when the charge is uniformly spread on a thin shell of radius $R$. Thus the internal energy of the object has the maximum $\epsilon_{\max }=E-\frac{1}{2} e^{2} / R$. If bound

(1.1) is valid at $e=0$, we expect the tighter entropy bound $S \leq(2 \pi R / \hbar)\left(E-\frac{1}{2} e^{2} / R\right)$ for the 
charged object. This coincides with Zaslavskii's proposal Eq.(1.2), and suggests its general validity.

Is Zaslavskii's proposed bound saturated by the Reissner-Nordström (RN) black hole ? Let the black hole's mass be $m$ and its charge $q$. If bound (1.2) applies to black holes, it predicts that

$$
S_{\mathrm{BH}} \leq \frac{2 \pi m}{\hbar}\left(m+\sqrt{m^{2}-q^{2}}\right)\left(1-\frac{q^{2}}{2 m\left(m+\sqrt{m^{2}-q^{2}}\right)}\right)
$$

[we set $E=m$ and $R=r_{+} \equiv m+\sqrt{m^{2}-q^{2}}$ ]. Multiplying out the factors we see that the right hand side is precisely $A / 4 \hbar$, where $A$ is the horizon area of the $\mathrm{RN}$ black hole,

$$
A=4 \pi r_{+}^{2}=4 \pi\left(m+\sqrt{m^{2}-q^{2}}\right)^{2}
$$

Thus the RN black hole saturates Zaslavskii's bound; this is a further point in favor of the bound's validity and efficiency. In Sec.V we shall arrive at a hybrid bound which embodies fully the requirement that the black hole be the most entropic state for a given quantity of energy, charge and angular momentum.

Nobody has thus far given a derivation of bound (11.2) for charged objects patterned after those originally used to derive bounds (1.1) and (1.5) from the GSL. Both those derivations focused on accretion of the relevant object by a black hole, and on the concomitant change in horizon area. Extension of this type of argument to the charged object is not straightforward. Suppose we work with a Schwarzschild black hole and a charged particle devoid of spin. Naively the particle's track is a geodesic, and so the minimal change in area will still be given by Eq.(1.3). In fact, if the black hole is a $\mathrm{KN}$ one, the same result is obtained by using Carter's integrals of motion for orbits of the Lorentz equation of motion [15]. Thus no improved entropy bound results for a nonrotating charged object. This is disturbing from the point of view of the derivation of entropy bounds by use of the GSL: if this approach is tenable, it should be possible to derive the physically reasonable bound (1.2) from the GSL once one accepts bound (1.1).

As we make clear in Sec.III, the mentioned problem may be traced to the neglect of a certain force that acts on the object. A charged particle in a black hole's vicinity is acted 
upon by not only the Lorentz force from the black hole's electromagnetic field, but also by the (Abraham-Lorentz-Dirac) radiation reaction force, as well as by the force originating from the black hole's polarization by the particle's electric field. Now it is known that a particle at rest in a static black hole background does not radiate (despite its being accelerated). Hence we expect the radiation reaction force to vanish. This suggests focusing on the accretion by a static black hole of an object which is lowered slowly from a large distance to the horizon. We can then suppose that only the gravitational, Coulomb and polarization (image) forces act upon it. By this approach we succeed below in deriving bound (1.2) by use of the GSL. Now, if as is sometimes claimed, the GSL functions independently of entropy bounds, there should not have been reason for an idiosyncratic effect (black hole polarization here) to supply precisely the missing element in the derivation of the entropy bound for charged objects from the GSL. This, to our mind, is the main significance of the mentioned success: a new demonstration that the GSL provides a valid road to entropy bounds.

\section{LOWERING A CHARGED BODY IN A BLACK HOLE'S FIELD}

We use the signature $\{-,+,+,+\}$ and denote the timelike coordinate outside the black hole, assumed to be a spherical static one, by $x^{0}$. First consider a test particle of mass $\mu$ and charge $e$. Its motion, were it subject only to gravitation and electromagnetic influences, would be governed by the lagrangian

$$
L=-\mu \int \sqrt{-g_{\alpha \beta} \dot{x}^{\alpha} \dot{x}^{\beta}} d \tau+e \int \hat{A}_{\alpha} \dot{x}^{\alpha} d \tau
$$

where $x^{\alpha}(\tau)$ denotes the particle's trajectory, $\tau$ the proper time, an overdot stands for $d / d \tau$, and $\hat{A}_{\alpha}$ means the background electromagnetic 4-potential evaluated at the particle's spacetime position. Recalling that $g_{\alpha \beta} \dot{x}^{\alpha} \dot{x}^{\beta}=-1$, it follows from the Lagrangian that the canonical momenta are $p_{\alpha}=\delta L / \delta \dot{x}^{\alpha}=\mu g_{\alpha \beta} \dot{x}^{\beta}+e \hat{A}_{\alpha}$. The stationarity of the envisaged background means there is a timelike Killing vector $\xi^{\alpha}=\{1,0,0,0\}$. The quantity

$$
\mathcal{E} \equiv-p_{\alpha} \xi^{\alpha}=-\mu g_{0 \beta} \dot{x}^{\beta}-e \hat{A}_{0}
$$


is easily shown to be conserved [18]; it corresponds to the usual notion of energy as measured at infinity. Its first term expands to $\mu+\frac{1}{2} \mu(d \mathbf{x} / d t)^{2}$ in the Newtonian limit. The second term, $-e \hat{A}_{0}$, is thus the electric potential energy.

In our gedanken experiment the object of rest mass $\mu$ and charge $e$, idealized as spherically symmetric, is suspended by some means to keep it from falling freely, and is slowly lowered radially towards the black hole. Of course, the forces keeping it quasistatic change its energy measured at infinity. The idea is to bring the object as close to the horizon as possible, and then drop it in, inferring from the energy measured at infinity at its last prefall position the increase in horizon area that this causes. A complication - the Unruh-Wald buoyancy in acceleration radiation [3] - may cause the object to float neutrally some distance from the horizon, thus arresting the contemplated descent. But as mentioned in Sec.I, provided the number of relevant particle species in nature is not large (which seems to be true in our universe), and provided the object is composed of parts that obey quantum mechanics, the buoyancy is negligible all the way to very near the horizon, and makes no practical difference to the energetics of the process (if the object is dropped from a bit off the horizon [4]).

For generality we allow the black hole to carry a charge $q$; we require that $q$ and the charge $e$ of the object be very small on the scale of $m$, the mass of the hole. There are now two parts to the electromagnetic potential: one linear in $q$, suitably named $\hat{A}_{0}^{(q)}$, which is produced by the black hole, and a second one, $A_{0}^{(e)}$, linear in $e$, whose source is the object itself. Because the last is a self-field, it has no hat symbol. As elucidated by Vilenkin 20 and corroborated by Smith and Will [21], by contrast to the situation in flat spacetime, in the presence of a Schwarzschild black hole this self-potential makes a nontrivial contribution to the object's energy measured at infinity. Those calculations are quite involved; here we recover that effect in a much simpler way. We may still write the energy as measured at infinity as in Eq.(3.2); however, we must there replace $\hat{A}_{0}$ by $\hat{A}_{0}^{(q)}+\frac{1}{2} A_{0}^{(e)}$. The factor $\frac{1}{2}$ is familiar from flat-spacetime electrodynamics; it takes care of the fact that the object owes part of its energy to its own field, not to the background one (we thank B. Linet for 
reminding us of this elementary fact). Smith and Will bring out the factor $\frac{1}{2}$ with an explicit calculation.

Correct to $O(e)$, which we regard as the same as $O(q)$, the metric may be taken as Schwarzschild's. In isotropic coordinates it is

$$
d s^{2}=-\left(\frac{1-\frac{m}{2 \rho}}{1+\frac{m}{2 \rho}}\right)^{2}\left(d x^{0}\right)^{2}+\left(1+\frac{m}{2 \rho}\right)^{4}\left[d \rho^{2}+\rho^{2}\left(d \theta^{2}+\sin ^{2} \theta d \phi^{2}\right)\right]
$$

We see that the horizon resides at $\rho=m / 2$. Because the object is nearly stationary, its 4 -velocity, which we normalize to -1 , must have the form $\dot{x}^{\alpha} \approx\left\{\left(-g_{00}\right)^{-1 / 2}, 0,0,0\right\}$. Substitution in Eq.(3.2) from the metric gives for the energy, when the object's CM is at $\rho=a$ and $\theta=0$,

$$
\mathcal{E}=\mu\left(1-\frac{m}{2 a}\right)\left(1+\frac{m}{2 a}\right)^{-1}-e\left(\hat{A}_{0}^{(q)}+\frac{1}{2} A_{0}^{(e)}\right)_{\rho=a, \theta=0}
$$

Eq. (A4) of the Appendix gives $A_{0}(\rho, \theta)$, the full electromagnetic 4-potential due to a stationary (or nearly so) point charge $e$ in the background of a spherical black hole with small charge $q$. This expression, accurate to $O\left(e^{2}\right)$, is a trivial extension of an early brilliant solution by Copson [22], as modified by Linet [23]. Its structure shows that one can think of the potential as getting a contribution from image charges on the black hole. $A_{0}(\rho, \theta)$ naturally diverges at $\rho=a$ and $\theta=0$, the charge's position. Thus if we want to use it for our finite object, we must regularize the potential before going to the limit $\rho \rightarrow a$ and $\theta \rightarrow 0$ as required by formula (3.4).

The simplest procedure is as follows. We reexpress $A_{0}$ in terms of new coordinates $\{\varrho, \vartheta, \phi\}$ centered on the charge, rather than on the black hole center, as was the case for $\{\rho, \theta, \phi\}$, but sharing the same polar axis. This implies the substitutions

$$
\begin{gathered}
\rho \cos \theta \rightarrow a+\varrho \cos \vartheta \\
\rho \rightarrow \sqrt{a^{2}+\varrho^{2}+2 a \varrho \cos \vartheta}
\end{gathered}
$$

A small metric sphere of proper radius $R$ located at $\rho=a$ and $\theta=0$ is the coordinate sphere $\varrho=\left(1+\frac{m}{2 a}\right)^{-2} R ; \forall \vartheta$. Thus it makes sense to expand $A_{0}$ in a Laurent series in $\varrho$ : 


$$
A_{0}=-\frac{\left(1-\frac{m}{2 a}\right)}{\left(1+\frac{m}{2 a}\right)^{3}} \frac{e}{\varrho}-4 a \frac{4 m a e+m^{2} q+4 a q(a+m)}{(2 a+m)^{4}}+C(a, m, e, q) \cos \vartheta+O(\varrho)
$$

where $C$ is a complicated function independent of $\varrho$.

Now the divergent term in Eq.(3.7) corresponds to the Coulomb potential of the charge $e$ in flat spacetime; there we expect $A_{0}=-e \varrho^{-1}$ (sign because we deal with the covariant component). A factor $\left(1-\frac{m}{2 a}\right)\left(1+\frac{m}{2 a}\right)^{-1}$ is required to redshift the fourth component of a 4 -vector to the point in question [see Eq.(3.3]. Finally, a factor $\left(1+\frac{m}{2 a}\right)^{-2}$ is required to convert the coordinate distance $\varrho$ in the denominator to proper distance. Thus when taking the limit $\rho \rightarrow a$ and $\theta \rightarrow 0(\varrho \rightarrow 0)$ of $A_{0}$, we must discard the first term in the r.h.s. of Eq.(3.7) (put another way, the energy that comes from it is absorbed into the renormalized rest mass $\mu$ [21).

Our spherically symmetric finite object samples all directions about its center without discrimination. Because the metric also looks isotropic in coordinates $\{\varrho, \vartheta, \phi\}$, we must thus average out the third term in the r.h.s. of Eq.(3.7) over all angles $\vartheta$ and $\phi$; as a result its contribution vanishes. Terms of $O(\varrho)$ vanish as the size of the object shrinks. Thus the second (constant) term in the r.h.s. of Eq.(3.7) furnishes the entire electrostatic contribution to $\mathcal{E}$. We separate that into the parts $A_{0}^{(q)}$ (black hole's) and $\hat{A}_{0}^{(e)}$ (image charges') defined earlier. Substituting these in Eq.(3.4) we find

$$
\mathcal{E}=\mu\left(1-\frac{m}{2 a}\right)\left(1+\frac{m}{2 a}\right)^{-1}+4 a e \frac{2 m a e+q(2 a+m)^{2}}{(2 a+m)^{4}}+O\left(\frac{e^{4}}{m^{3}}\right)
$$

The case $q=0$ of this result is equivalent to results given earlier (in Schwarzschild coordinates) by Vilenkin [20] for $a \gg m$ and by Smith and Will [21] for all $a$.

When the object is near the horizon, the proper distance from its CM to the horizon is

$$
\ell \equiv \int_{m / 2}^{a}\left(g_{\rho \rho}\right)^{1 / 2} d \rho \approx 4(a-m / 2)+O\left[(a-m / 2)^{2}\right]
$$

Expressing $a$ in Eq.(3.8) in terms of $\ell$ by means of Eq.(3.9) we get

$$
\mathcal{E}=\left(\frac{2 \mu \ell+e^{2}}{8 m}\right)\left[1+O\left(\frac{\ell}{m}\right)\right]+\frac{e q}{2 m}+O\left(\frac{e^{4}}{m^{3}}\right)
$$


Since we are obviously considering a black hole large and massive compared to the object's proper radius and mass, the corrections of $O(\ell / m)$ are appropriately neglected, as are those of $O\left(e^{4} / m^{3}\right)$ by virtue of the assumed smallness of $e$. The gradual approach to the horizon must stop when the proper distance from the object's CM to the horizon reaches the object's proper radius $R$. Hence,

$$
\mathcal{E} \geq \frac{2 \mu R+e^{2}+4 e q}{8 m}
$$

As mentioned, our primary concern is with changes in the horizon area. Although we have used Schwarzschild's metric in the above discussion, the true metric is closer to that of a RN solution; it is thus best to use as a first approximation the area appropriate to the RN black hole, namely Eq.(2.3). This formula must be corrected for the perturbation of the metric originating in the object, which in linear approximation should be of $O(\mu)$ and $O\left(e^{2}\right)$, the first caused by the energy momentum tensor of the object's mass, and the second by the overall electromagnetic energy momentum tensor [recall that we take $O(e)=O(q)$ ]. We now argue that the corrections to the area formula actually appear only in the next higher orders.

For suppose the area $A$ were indeed perturbed in linear approximation to $O(\mu)$ and $O\left(e^{2}\right)$. By spherical symmetry of the background these corrections would not depend on the direction along which the object was lowered. If $n$ equal bodies were lowered, each along a different radial direction, the perturbation would be $n$ times larger by linearity of the approximation. But if enough bodies were disposed on a spherical shell concentric with the black hole, the perturbation of the metric at the horizon should tend to zero by Birkhoff's theorem [18] that the metric exterior to a spherical charged black hole is exactly RN if the surroundings are spherically symmetric too. We thus get a contradiction unless we admit that the perturbations of $O(\mu)$ and $O\left(e^{2}\right)$ vanish in linear theory. Any corrections to $A$ must be of higher order, like $O\left(\mu^{2}\right)$, etc. Hence by Eq.(2.3)

$$
A=8 \pi\left(2 m^{2}-q^{2}\right)+O\left(q^{4} / m^{2}\right)+O\left(\mu^{2}\right)+O\left(\mu e^{2} / m\right)
$$


where we have included all possible second order terms of the correct dimensions; $O\left(e^{4} / m^{2}\right)$ is subsummed in the $O\left(q^{4} / m^{2}\right)$ which is the remainder of the expansion of $A$ in powers of q. Below we denote the above sort of corrections by the ellipsis $\cdots$.

The descent of the object, if sufficient slow, is known to be an adiabatic process which causes no change in the horizon area 24, 25. It follows that to the stated accuracy, $m$ is unchanged in the course of the lowering process itself because $q$ and $A$ are unchanged. When the object is finally absorbed by the black hole, $m$ increases by $\mathcal{E}$ while $q$ is augmented by $e$; after the suspension machinery has been withdrawn (if adiabatically done, this will cause no further area increase 24,25$]$, we get an unperturbed RN black hole with mass $m+\mathcal{E}$ and charge $q+e$.

Calculating its horizon area from Eq.(3.12) and substracting the area of what was at first an unperturbed RN black hole of mass $m$ and charge $q$ (because $e$ was still distant), we find the change

$$
\Delta A=8 \pi\left(4 m \mathcal{E}-2 q e-e^{2}\right)+O\left(\mathcal{E}^{2}\right)+\cdots
$$

Finally substitution of Eq.(3.11) gives

$$
\Delta A \geq\left(8 \pi \mu R-4 \pi e^{2}\right)\left[1+O\left(\frac{\mu R}{m^{2}}\right)\right]+\cdots
$$

Notice that the black hole parameters $m$ and $q$ have dropped out from the dominant terms, in analogy with results for uncharged [14] or spinning [13] objects. The minimum change in black hole entropy, $\Delta A / 4 \hbar$ corresponding to the equality in (3.14), is thus a property of the object itself. The entropy of the object cannot exceed this amount, lest the overall entropy of the world decrease upon the object's assimilation. We thus find the bound on the entropy of an object of charge $e$, proper energy $E=\mu$ and radius $R$ to coincide with Zaslavskii's proposal Eq.(1.2). 


\section{VARIANT EMPLOYING A GROUNDED BLACK HOLE}

In Sec.III the black hole is electrically isolated so its charge $q$ is fixed. One can consider a variant gedanken experiment involving a black hole which is electrically grounded. An approximation of this could be achieved by having a conducting "wire" connect the horizon to matter at large distances. One wonders whether a different, perhaps tighter, bound on entropy would be obtained from a repetition of the above gedanken experiment. Here we show that the same bound arises despite the differences in the energetics.

Eq. A5) in the Appendix gives the potential $A_{0}^{(g)}(\rho, \theta)$ engendered by a charge $e$ located at $\rho=a$ and $\theta=0$ in the vicinity of a grounded spherical static black hole. This potential vanishes both at infinity and at the horizon $(\rho=m / 2)$, and may be obtained by setting

$$
q=-8 a m e(2 a+m)^{-2}
$$

in the expression for $A_{0}(\rho, \theta)$, Eq. (A4). Although now the hole's charge (aside from the image charge) is controlled by the external charge's position, the contribution of $q$ to energy is still lumped with the black hole (see below). Therefore, $q$ is still regarded as generating an external potential for $e$. We may thus take over Eq.(3.8) for the object's energy, but with $q$ replaced according to Eq.4.1).

Expression (3.10) for $\mathcal{E}$ is thereby replaced by

$$
\mathcal{E}=\left(\frac{2 \mu \ell-3 e^{2}}{8 m}\right)\left[1+O\left(\frac{\ell}{m}\right)\right]+O\left(\frac{e^{4}}{m^{3}}\right)
$$

Neglecting the corrections we get in place of Eq.(3.11) the very different result

$$
\mathcal{E} \geq \frac{2 \mu R-3 e^{2}}{8 m}
$$

Unlike the case discussed in Sec.III, here $\mathcal{E}$ is not the exclusive contribution to the change in $m$. When the object with charge $e$ is very distant from it, the black hole is exactly Schwarzschild [because $q=0$ by Eq.(4.1)] with mass $m$ and horizon area $16 \pi m^{2}$. As the object approaches, charge flows into the hole through the wire, and $q$ varies according to 
Eq.(4.1). Because the descent is slow, the change in the hole is adiabatic; thus it should not cause growth of the horizon's area [24,25]. We shall assume the current flows reversibly (in the sense of Christodoulou's transformations [16]), so that it does not cause a change of area either. According to Eq.(3.12), $m$ will have to grow to compensate for the increase in $q$. In the limit $a \rightarrow m / 2, q \rightarrow-e$ so that $m \rightarrow m^{\prime} \equiv \sqrt{m^{2}+e^{2} / 2} \approx m+e^{2} / 4 m$. When the object is assimilated, its charge $e$ exactly neutralizes the hole's charge, while its energy augments the hole's mass $m^{\prime}$ to $m^{\prime \prime}=m+e^{2} / 4 m+\mathcal{E}$. The new horizon area is thus $16 \pi\left(m+e^{2} / 4 m+\mathcal{E}\right)^{2}$. Substituting $\mathcal{E}$ from Eq. (4.3), and substracting $16 \pi m^{2}$ we obtain the overall change in area

$$
\Delta A \geq 8 \pi \mu R\left[1+O\left(\frac{\mu R}{m^{2}}\right)\right]-4 \pi e^{2}+\cdots
$$

This is the same as Eq.(3.14), so that from its minimum value we reproduce the bound on entropy of a charged object, Eq.(1.2).

How do we get the same area increase out of two different expressions for the energy of

the object, (3.10) and (4.2) ? The difference is compensated by a complementary difference in the behavior of the electric potential of the horizon. In the first case as $a \rightarrow m / 2$, $A_{0} \rightarrow-(e+q) / 2 m$. In the second case $A_{0}^{(g)}(m / 2, \theta)=0$ identically. Thus in the first case the infall of the charge contributes a change in horizon area through the change in black hole charge, while in the second it does not.

\section{THE OPTIMAL BOUND}

For an object with spin $s$, charge $e$, maximal radius $R$ and mass-energy $E=\mu$ we may, by comparing Eqs.(1.2) and (1.5), conjecture the tighter entropy bound

$$
S \leq 2 \pi \frac{\sqrt{E^{2} R^{2}-s^{2}}-e^{2} / 2}{\hbar}
$$

As a check we look at the case of slow rotation:

$$
S \leq \frac{2 \pi R}{\hbar}\left[E-\frac{s^{2}}{2 \mu R^{2}}-\frac{e^{2}}{2 R}\right]+O\left(s^{4}\right)
$$


Comparing with remarks made in Sec.II we see that here the maximum possible Coulomb energy and $\frac{2}{3}$ of the maximum possible rotational energy are deducted from the total energy, with the remainder taken as the $E$ in the original entropy bound (1.1). Obviously, for a nonrelativistically rotating ordinary object, bound (5.2) is correct, and on the liberal side.

This correspondence argument does not prove the correctness of bound (5.1); that bound is not the unique progenitor of the nonrelativistic form (5.2). In addition, one could argue that there seems to be something missing from Eq.(5.1). A spinning charged object has a magnetic dipole moment proportional of $O(e s / \mu)$ which generates a magnetic field, and thus contributes to the electromagnetic energy. We see no such contribution reflected in bound (5.2). However, it must be recalled that magnetic dipole energy is of higher order in $c^{-1}$ than Coulomb energy. If we care about this higher order, we should continue the Taylor expansion of the root in (5.1) to $O\left(s^{4}\right)$ which is of the same order. However, we have just mentioned that bound (5.2) understates the amount of rotational energy in the system by a substantial factor already at $O\left(s^{2}\right)$. It is thus pointless to go to higher order in rotational or electromagnetic energy. Bound (5.2) is not strict, but liberal, and so is bound (5.1). Thus at present we find no reason to cast doubt on the general bound (5.1).

A more positive point for bound (5.1) is the fact that any KN black hole (mass $m$, charge $q$ and angular momentum $j$ ) saturates it. The horizon area of such black hole is [18]

$$
A=4 \pi\left(r_{+}^{2}+j^{2} / m^{2}\right) ; \quad r_{+} \equiv m+\left(m^{2}-j^{2} / m^{2}-q^{2}\right)^{1 / 2}
$$

Substituting $r_{+}$, squaring as required, and cancelling terms gives

$$
A=2 \pi\left(4 m r_{+}-q^{2}\right)=2 \pi\left[4 m\left(r_{+}^{2}+j^{2} / m^{2}-j^{2} / m^{2}\right)^{1 / 2}-q^{2}\right]
$$

In light of Eq.(5.3) it is reasonable interpret $\left(r_{+}^{2}+j^{2} / m^{2}\right)^{1 / 2}$ as the radius $R$ of the hole. Incorporating this in the last equation and dividing by $4 \hbar$ gives for the black hole entropy

$$
S_{\mathrm{BH}}=\frac{2 \pi}{\hbar}\left[\left(m^{2} R^{2}-j^{2}\right)^{1 / 2}-q^{2} / 2\right]
$$

If we identify $m \leftrightarrow E, q \leftrightarrow e$ and $j \leftrightarrow s$, this is exactly the upper bound of Eq.(5.1). Hence the KN black hole saturates the proposed entropy bound. This property would be lost if 
modifications were made to the bound. Hence we adopt it in the form given. Study of the role of spin-curvature effects in the discussion in Sec.III is in progress in order to provide a more direct argument for the full bound (5.1).

Parenthetically we should mention another way to look at the saturation. Suppose we had some means to slowly lower a small KN black hole with mass $\mu$, charge $e$ and angular momentum $s$ into a much larger KN black hole with corresponding parameters $m, q$ and j. Then the black holes would merge reversibly, i.e., with no overall growth in horizon area. This is obvious because if bound (5.1) can be derived by the arguments expounded in Secs.III and IV, then the overall growth in area of the big black hole must correspond precisely to the equality case in bound (5.1) (for parameters $\mu, e$ and $s$ ) multiplied by $4 \hbar$. But this just says that the big horizon expands by precisely the area of the small horizon, so that the merged horizon has area equal to the sum of the two original ones.

Bound (5.1) is readily generalized to include magnetic monopole charge $g$. Duality of electromagnetism leaves little doubt that one should just replace $e^{2} \rightarrow e^{2}+g^{2}$. The deeper question arises, can one give generic bounds on entropy which are tighter than (5.1) by virtue of the object possessing some conserved "quantum number" apart from $q, g$ or $s$ ? A case in point would be a tighter bound for an object with definite and known baryon number. We now marshal evidence in support of the conjecture that bound (5.1), with the extension to magnetic monopole, cannot be bettered generically. By "generically" we mean without knowledge of details about the object's structure and dynamics. When these are known it is possible to compute by means of statistical mechanics bounds on the entropy which can be small compared to bound (1.1), for example [6]. But if we use no such information, we must go back to the black hole derivation of the entropy bounds, and it is for this situation that we conjecture that bound (5.1) cannot be bettered.

The "no hair" conjecture is central to our argument. A large amount of work has certified that a stationary black hole can have just a few parameters. The incontestable ones are mass, charge, magnetic monopole and angular momentum. Skyrmion number is an extra possibility [26], but one whose physical significance is unclear [15]. Other candidates, 
such as color [27], scalar charge [28] and massive Yang-Mills charge [29], are associated with unstable black holes [30]. The sort of arguments we have given in Secs.III and IV make sense only if the black hole is stable to outside perturbations. Hence we focus on the KN black holes with parameters $m, q, g$ and $j$.

Suppose we add to such a black hole an object carrying an extra additive conserved quantity $b$. If $b$ is a "global" quantity, such as baryon or lepton numbers are thought to be, it generates no field of its own. The energy-momentum tensor originating in the object is thus unaffected by $b$ (put another way, effects of $b$ can be absorbed in the mass). Hence, even if perturbations of the metric are taken into account, $b$ cannot directly perturb the horizon area formula (5.3), and so $m$ is unaffected by slow (adiabatic) lowering of the object. Further, absence of $b$ from the list of black hole parameters means the black hole has no chemical potential conjugate to $b$. Thus when the object finally enters the hole, it cannot change the horizon area except through the change in $m$, which is $\mathcal{E}$. But $\mathcal{E}$ gets no contribution specific to $b$ since the latter does not generate a field that could polarize the hole, c.f. Eq.(3.2). Therefore, the change in horizon area is $b$-independent. But then the bound that can be set on the entropy by the argument of Sec.III is also independent of $b$ : the new quantity does not allow tightening of the entropy bound.

Much the same conclusion can be reached if $b$ generates a short range field, schematically denoted by $\mathcal{B}$. For example, $b$ could be weak hypercharge, a source of the short range, $Z_{-}^{-}$ boson mediated, weak force. Although there is now a contribution to the energy-momentum tensor from $\mathcal{B}$, it is localized around the object, and thus can be lumped into its usual energymomentum tensor. No novel perturbation to the metric arises from this. Hence, $b$ cannot directly perturb the horizon area formula (5.3), and so $m$ is unaffected by slow lowering of the object. Furthermore, no novel potential term is contributed to $\mathcal{E}$ by $\mathcal{B}$ unless the particle is already next to the horizon; otherwise the short range field $\mathcal{B}$ does not reach down to the horizon and cannot polarize it. Hence, in this case also, the change in horizon area turns out to be $b$-independent, and $b$ cannot appear in a generic entropy bound.

The third and last case is when $b$ is the source of a long-range field, again denoted $\mathcal{B}$. 
The range may be finite if large compared to typical object size. Now the area formula may differ from Eq. (5.3) by terms depending on $b$ because of the perturbation that $\mathcal{B}$ 's energy momentum tensor exerts on the metric. Unless $\mathcal{B}$ is a gauge field which (unlike the $Z$ and $W$ boson fields of Weinberg-Salam theory) remains massless in the classical (or low energy) limit, we cannot rule out such dependence, as we did in Sec.III. This is because Birkhoff-type theorems exist only for massless vector fields, and from our point of view the electromagnetic field is the only one such, and has already been accounted for in Secs.III and IV. Thus, while the area stays constant during the descent as required by the adiabatic theorem, $m$ may change by a quantity of $O\left(b^{2}\right)$ as the object descends. The sign of this quantity is unclear without a specific model.

In addition, $\mathcal{E}$ is most likely to have a term of $O\left(b^{2}\right)$ for the same reasons as in Eq.(3.10) (by "no hair" there is now no analog of $q$ ). It may even be that this term is also positive here, yet it does not follow that the effect of $b$ is to suppress the growth of area, as it did in Sec.III, because of the correction of indefinite sign to the area formula. Thus without calculating linear corrections to the metric, one cannot settle the question of whether the change in area is incremented or depressed by $b$ 's presence. However, we have as yet uncovered no

clear evidence that an improved bound (5.1) will result for a long-range field which is not a massless vector field. The conjecture that bound (5.1) is the tightest generic bound on entropy thus seems reasonable.

ACKNOWLEDGMENTS We thank Shahar Hod for discussions, and Bernard Linet for pointing out a crucial omission. This research is supported by a grant from the Israel Science Foundation, established by the Israel Academy of Sciences and Humanities.

\section{APPENDIX A: COPSON-LINET SOLUTION FOR CHARGE IN BLACK HOLE BACKGROUND}

Here we determine $A_{0}$ resulting from a charge $e$ in the Schwarzschild background (3.3). Using the conventions of Misner, Thorne and Wheeler [18] we write the electromagnetic field 
as $F_{\alpha \beta}=A_{\beta, \alpha}-A_{\alpha, \beta}$. We express the Maxwell equations $F_{; \beta}^{\alpha \beta}=4 \pi j^{\alpha}$ for the axisymmetric stationary field of a test point charge $e$ situated at $\{\rho, \theta\}=\{a, 0\}$ as

$$
\left[\frac{\rho^{2} A_{0, \rho}}{\left(1-\frac{m}{2 \rho}\right)\left(1+\frac{m}{2 \rho}\right)^{2}}\right]_{, \rho}+\frac{A_{0, \theta \theta}}{\left(1-\frac{m}{2 \rho}\right)\left(1+\frac{m}{2 \rho}\right)^{2}}=4 \pi e \delta(\rho-a) \delta(\theta) \delta(\phi)
$$

The source term takes on the indicated form because the relevant 3-D Dirac delta function has the form $(-g)^{-1 / 2} \delta(\rho-a) \delta(\theta) \delta(\phi)$.

A convenient solution of this equation was found by Copson [22] long ago:

$$
A_{0}^{(\mathrm{C})}(\rho, \theta)=-e \frac{\chi+\frac{m^{2}}{4 a^{2} \chi}}{\left(1+\frac{m}{2 \rho}\right)^{2} \rho\left(1+\frac{m}{2 a}\right)^{2}}
$$

where

$$
\chi(\rho, \theta) \equiv\left[\frac{\rho^{2}+\left(m^{2} / 4 a\right)^{2}-2\left(m^{2} / 4 a\right) \rho \cos \theta}{\rho^{2}+a^{2}-2 a \rho \cos \theta}\right]^{1 / 2}
$$

In $A_{0}^{(\mathrm{C})}$ there appears in denominators not only the Euclidean distance $\left(\rho^{2}+a^{2}-2 a \rho \cos \theta\right)^{1 / 2}$ between the field point $\{\rho, \theta\}$ and the charge's position, but also the distance of the former from the point $\left\{m^{2} / 4 a, 0\right\}$, which is the appropriate location for the image charge in the solution of Laplace's equation for a charge near a conducting sphere of radius $m / 2$ by the method of images. This is consistent with the expectation that the black hole is polarized by influence of the charge $e$.

As noted by Linet [23], the coefficient of the $1 / \rho$ term in the asymptotic form of this potential indicates that a total of charge $e-e(m / a)(1+m / 2 a)^{-2}$ resides in the spacetime. The charge $e$ being the only source outside the black hole, one must perforce admit that the black hole bears charge $\tilde{e}=-e(m / a)(1+m / 2 a)^{-2}$. Of course such charge must modify the metric, as does the exterior charge $e$. But such perturbations will be of order $e^{2}$ and may be ignored in computing $A_{0}^{(\mathrm{C})}$ correct to $O\left(e^{2}\right)$. Linet proposes that a more relevant solution to the problem is to be had by adding to $A_{0}^{(\mathrm{C})}$ a monopole field with charge $-\tilde{e}$. We shall push this a little farther and add to $A_{0}^{(\mathrm{C})}$ the monopole potential appropriate to charge $q-\tilde{e}$. Since the spherically symmetric, everywhere regular, solution of Eq.(A1) is $\rho^{-1}\left(1+\frac{m}{2 \rho}\right)^{-2}$, we must write 


$$
A_{0}(\rho, \theta)=A_{0}^{(\mathrm{C})}(\rho, \theta)-\frac{q+4 \frac{e m a}{(2 a+m)^{2}}}{\rho\left(1+\frac{m}{2 \rho}\right)^{2}}
$$

The charge in the spacetime is now $q+e$, with $q$ in the black hole, as it should. So long as $q$ is of order $e$ and this last is small on the scale of $m$, we do not have to correct the metric or Eq.(A1) to get $A_{0}$ correct to $O\left(e^{2}\right)$.

Potential (A4) has the constant value $-q / 2 m-4 a e(2 a+m)^{-2}$ on the horizon $(\rho=m / 2)$. One can ask the question, what would be the potential if the charge $e$ were to coexist with a black hole which is grounded. In practice this could be achieved by having a conductor connect the horizon to matter at large distances. The desired solution of Eq. (A1) is now one satisfying $A_{0}(m / 2, \theta)=A_{0}(\infty, 0)=0$. It is easily checked that the desired potential can be gotten from $A_{0}$ in Eq. (A4) by setting $q \rightarrow-8 a m e(2 a+m)^{-2}$. We denote it by $A_{0}^{(g)}$ ("g" for "grounded"):

$$
A_{0}^{(g)}(\rho, \theta)=A_{0}^{(\mathrm{C})}(\rho, \theta)+4 \frac{\frac{e m a}{(2 a+m)^{2}}}{\rho\left(1+\frac{m}{2 \rho}\right)^{2}}
$$

Of course, in the present case the charge on the black hole varies with $a$; this is because as the charge $e$ draws near the black hole, opposite charges are drawn into the hole through the conductor.

[1] J. D. Bekenstein, Phys. Rev. D23, 287 (1981).

[2] J. D. Bekenstein Phys. Rev. D9, 3292 (1974).

[3] W. G. Unruh and R. M. Wald, Phys. Rev. D25, 942 (1982)

[4] J. D. Bekenstein, Phys. Rev. D49, 1912 (1994).

[5] M. A. Pelath and R. M. Wald, gr-qc/9901032.

[6] J. D. Bekenstein, Phys. Rev. D30, 1669 (1984).

[7] J. D. Bekenstein and M. Schiffer, Phys. Rev D39, 1109 (1989). 
[8] J. D. Bekenstein and M. Schiffer, Int. J. Mod. Phys. C 1, 355 (1990).

[9] O. Zaslavskii, Class. Quant. Gravity 13, L7 (1996).

[10] R. D. Sorkin, R. M. Wald and Z. Z. Jiu, Gen. Rel. Grav. 13, 1127 (1981).

[11] O.Zaslavskii, Phys. Lett. A160, 339 (1991).

[12] O. Zaslavskii, Gen. Rel. Grav. 24, 973 (1992).

[13] S. Hod, gr-qc/9901035.

[14] J. D. Bekenstein, Phys. Rev. D7, 2333 (1973).

[15] J. D. Bekenstein, gr-qc/9808028.

[16] D. Christodoulou, Phys. Rev. Lett. 25, 1596 (1970).

[17] B. Carter, Phys. Rev. D 174, 1559 (1968).

[18] C. W. Misner, K. S. Thorne and J. A. Wheeler, Gravitation (Freeman, San Francisco, 1973)

[19] R. Hojman and S. Hojman, Phys. Rev. D15, 2724 (1977).

[20] A. Vilenkin, Phys. Rev. D 20, 373 (1979).

[21] A. G. Smith and C. M. Will, Phys. Rev. D 22, 1276 (1980).

[22] E. Copson, Proc. Roy. Soc. London A118, 184 (1928).

[23] B. Linet, J. Phys. A9, 1081 (1976).

[24] J. D. Bekenstein, in Black Holes, Gravitational Radiation and the Universe, eds B. R. Iyer and B. Bhawal (Kluwer, Dordrecht 1998).

[25] A. E. Mayo, Phys. Rev. D58, 10400 (1998).

[26] S. Droz, M. Heusler and N. Straumann, Phys. Lett. B268, 371 (1991); M. Heusler, N. Straumann and Z. H Zhou, Helv. Phys. Acta 66614 (1993). 
[27] M. S. Volkov and D. V. Gal'tsov, JETP Lett. 50, 312 (1989) and JETP Lett. 50, 346 (1990); P. Bizon, Phys. Rev. Lett. 64, 2844, 1990.

[28] N. Bocharova, K. Bronnikov and V. Melnikov, Vestn. Mosk. Univ. Fiz. Astron. 6, 706 (1970); J. D. Bekenstein, Ann. Phys. (NY) 82, 535 (1974) and 91, 72 (1975).

[29] B. R. Greene, S. D. Mathur and C. M. O’Neill, Phys. Rev. D 47, 2242 (1993).

[30] O. Brodbeck and N. Straumann, Phys. Lett. B324, 309 (1996) and J. Math. Phys. 37, 1414 (1994); N. E. Mavromatos and E. Winstanley, Phys. Rev. D53, 3190 (1996); N. Straumann and Z. H. Zhou, Phys. Lett.B243, 33 (1991) and Nucl. Phys. B369, 180 (1991); K. A. Bronnikov and Yu. N. Kireyev, Phys. Lett. A67, 95 (1978). 